before culture being about 20 hours. Tissues from young individuals yield significantly more cells per gram and these cells have a higher proliferative capacity. Intrinsic differences in growth potential and/or lineage potential may affect the utility of cell transplantation or other applications for therapy. Questions relating to the in vitro lifespan of these cells and the role of telomerase ${ }^{10}$, as well as the importance of DNA methylation, still need to be addressed.

Using cells cultured from embryonic tissues bypasses some of these concerns, but also raises complex ethical and societal issues. Careful evaluation and consideration of the relative merits of post-mortem or adultderived cells and fetal progenitor cells will be necessary.

Theo D. Palmer ${ }^{\star} \uparrow$, Philip H. Schwartz

Philippe Taupin*, Brian Kaspar*,

Stuart A. Stein $\ddagger$, Fred H. Gage ${ }^{\star}$

${ }^{*}$ The Salk Institute, Laboratory of Genetics, 10010

North Torrey Pines Road, La Jolla, California 92122,

USA

e-mail: gage@salk.edu

$\dagger$ Stanford University, Department of Neurosurgery,

MSLS P304, 1201 Welch Road,

Stanford, California 94305-5487, USA

$\ddagger$ Children’s Hospital of Orange County, Brain and

Tissue Bank for Developmental Disorders, 455 South

Main Street, Orange, California 92868, USA

1. Palmer, T. D., Markakis, E. A., Willhoite, A. R., Safar, F. \& Gage, F. H. J. Neurosci. 19, 8487-8497 (1999).

2. Reynolds, B. A. \& Weiss, S. Science 255, 1707-1710 (1992).

3. Svendsen, C. N., Caldwell, M. A. \& Ostenfeld, T. Brain Pathol. 9, 499-513 (1999).

4. Fricker, R. A. et al. J. Neurosci. 19, 5990-6005 (1999).

5. Pincus, D. W. et al. Ann. Neurol. 43, 576-585 (1998).

6. Johansson, C. B., Svensson, M., Wallstedt, L., Janson, A. M. \& Frisen, J. Exp. Cell Res. 253, 733-736 (1999).

Pagano, S. F. et al. Stem Cells 18, 295-300 (2000).

8. Roy, N. S. et al. Nature Med. 6, 271-277 (2000).

Taupin, P. et al. Neuron 28, 385-397 (2000).

10. Ostenfeld, T. et al. Exp. Neurol. 164, 215-226 (2000).

\section{Boundary effects}

\section{Refraction of a particle beam}

- he refraction of light at an interface is familiar as a rainbow or the 'bending' of a pencil in a glass of water. Here we show that particles can also be refracted and even totally internally reflected, as evidenced by an electron beam of $28.5 \times 10^{9}$ electron volts being deflected by more than a milliradian upon exiting a passive boundary between a plasma and a gas - the electron beam is bent away from the normal to the interface, just like light leaving a medium of higher refractive index. This phenomenon could lead to the replacement of magnetic kickers by fast optical kickers in particle accelerators, for example, or to compact magnet-less storage rings in which beams are guided by plasma fibre optics.

Refraction is caused by electrostatic plasma fields set up when plasma electrons are

Figure 1 Experimental and simulation results demonstrating refraction of an electron beam at a plasma-gas interface. a, Actual electron-beam deflection (circles), measured using a beam-position monitor, and the theoretical deflection (blue line) as a function of the incident angle. $\mathbf{b}$, Simulation: perspective image of a beam emerging from plasma (turquoise); the inward motion of the plasma electrons is visible as a depression in the plasma surface behind the beam. c, Experiment: image of the beam downstream of the plasma, showing the deflected beam and the undeflected transient (at the crosshairs); $\mathbf{d}$, head-on view of image in $\mathbf{b}$. The beam consisted of $1.9 \times 10^{10}$ electrons at $28.5 \mathrm{GeV}$ in a gaussian bunch of length $\sigma_{\mathrm{z}}=0.7 \mathrm{~mm}$ and spot size $\sigma_{\mathrm{x}} \approx \sigma_{\mathrm{y}} \approx 40 \mu \mathrm{m}$. The plas$\mathrm{ma}$, with radius $2.3 \mathrm{~mm}$, length $1.4 \mathrm{~m}$
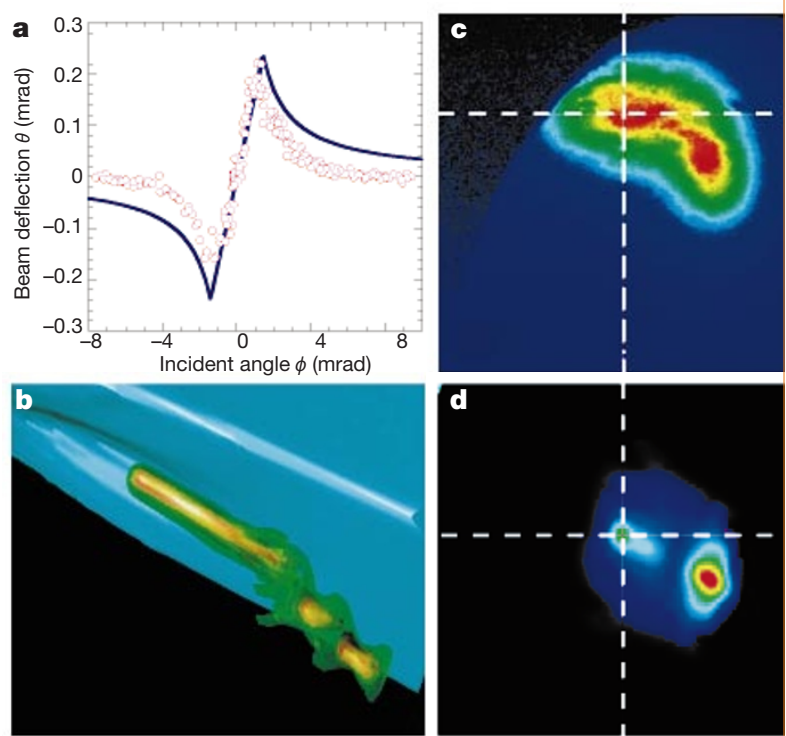
and density $1 \times 10^{14} \mathrm{~cm}^{-3}$, was created by photoionization of lithium vapour by an ArF laser. The angle, $\phi$, between the electrons' initial trajectory and the plasma boundary was controlled by adjusting the tilt angle of the final laser-beam mirror.

expelled by the collective space charge force of the head of the beam. The plasma ions in the beam path are more massive and remain, constituting a positively charged channel through which the latter part of the beam travels. The ions provide a net force that focuses the beam ${ }^{1,2}$. When the beam comes close to the plasma boundary, the ion channel becomes asymmetric, producing a deflecting force in addition to the focusing force. This formation of an asymmetric plasma lens ${ }^{3}$ gives rise to the bending of the beam path at the interface.

The order of magnitude of this deflection can be estimated, yielding an expression for the deflection angle, $\theta$, as a function of the incident angle, $\phi$. This is the effective nonlinear Snell's law for the electron beam refraction, valid for $\phi$ greater than $\theta$ :

$$
\theta=\left(8 \propto N r_{\mathrm{e}}\right) /\left(\pi \sqrt{2 \pi} \gamma \sigma_{\mathrm{z}} \sin \phi\right)
$$

where $N / \sqrt{2} \pi \sigma_{z}$ is the charge per unit length of the beam, $r_{\mathrm{e}}$ is the classical electron radius, $\gamma$ is the beam's energy in units of $m c^{2}$ and $\alpha$ is a factor of order one that is a weak function of plasma density and bunch length. When $\phi$ is less than $\theta$, this equation breaks down and the beam is internally reflected. Simulations ${ }^{4}$ show that $\theta \approx \phi$ (critical reflection) for small values of $\phi$.

We tested this analytical model by using the electron beam at the Stanford Linear Accelerator Center (Final Focus test facility), as described ${ }^{5,6}$. Sample results are shown in Fig. 1 and compared with a full three-dimensional electromagnetic particle-in-cell computer simulation? ${ }^{7}$ In Fig. 1a, the solid curve represents the prediction from the model (with $\alpha=0.2$ ): for incident angles smaller than $1.3 \mathrm{mrad}$, the beam appears to be internally reflected, in agreement with the model.

Figure $1 \mathrm{~b}$ shows a snapshot of the real space of the beam and plasma electron density (turquoise) from a simulation. A transient at the head of the beam is apparent because of the finite time that it takes the plasma to respond to the beam. The tail portion is deflected towards the plasma and is near the plasma boundary. The transient results in the characteristic splitting of the beam images downstream, as shown in Fig. 1c, d.

The simulations and experimental results presented here show that it is possible to refract and even reflect a particle beam from a dilute plasma gas. Remarkably, for a 28.5$\mathrm{GeV}$ beam that can bore through several millimetres of steel, the collective effects of a plasma are strong enough to 'bounce' the beam off an interface that is one million times less dense than air.

Patric Muggli ${ }^{\star}$, Seung Lee ${ }^{\star}$, Thomas Katsouleas ${ }^{\star}$, Ralph Assmann $\dagger$, Franz-Joseph Decker $\dagger$, Mark J. Hogan $\dagger$, Richard Iverson $\dagger$, Pantaleo Raimondi $\dagger$, Robert H. Siemann $\dagger$, Dieter Walz†, Brent Blueł, Christopher E. Clayton $\ddagger$, Evan Dodd $\ddagger$ Ricardo A. Fonseca $\ddagger$,

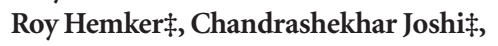
Kenneth A. Marsh $\ddagger$, Warren B. Morił, Shoquin Wang $\ddagger$

${ }^{*}$ University of Southern California, Los Angeles, California 90089, USA

e-mail: katsoule@usc.edu

$\uparrow$ Stanford Linear Accelerator Center, Stanford

University, Stanford, California 94309, USA

\$University of California, Los Angeles, California 90095, USA

1. Su, J. J., Katsouleas, T. \& Dawson, J. M. Phys. Rev. A 41, 3321-3331 (1990).

2. Whittum, D., Sessler, A. \& Dawson, J. M. Phys. Rev. Lett. 64, 2511-2514 (1990)

3. Chen, P. Part. Accel. 20, 171-182 (1987).

4. Katsouleas, T. et al. Nucl. Instrum. Meth. Phys. Res. A 455, 161-165 (2000)

5. Muggli, P. et al. Phys. Rev. Sp. Top. AB (submitted)

6. Hogan, M. J. et al. Phys. Plasmas 7, 2241-2248 (2000).

7. Hemker, R., Mori, W. B., Lee. S. \& Katsouleas, T. Phys. Rev. Sp. Top. AB 3, 61301-61305 (2000). 